\title{
Research on the "Digital Divide" and Social Integration of the Elderly in the Post-epidemic Era
}

\author{
Yanyan $\mathrm{Zhu}{ }^{1, *}$,Weifeng $\mathrm{Li}^{2}$ \\ ${ }^{1,2}$ School of Political Science and Law, University of Jinan, Jinan, Shandong 250022, China \\ *Corresponding author. Email: 1782231380@qq.com
}

\begin{abstract}
At present, the Internet participation of the elderly is showing a rapid rising trend. Since the outbreak of COVID-19, the news of the elderly being hindered from traveling without health code has attracted wide attention. With the rapid development of digital information, the phenomenon of "digital divide" of aging population has also emerged. Based on the current situation of epidemic prevention and control and population aging, this paper puts forward several views on the "digital divide" and social integration of the elderly group.
\end{abstract}

Keywords: Post-epidemic era, The elderly, Digital divide, Social integration

\section{INTRODUCTION}

With the continuous improvement of science and technology and the deepening of the degree of aging, China has entered the information age. The Internet has greatly changed the way people live, study and work, and has had a profound impact on society and economy.

In novel virus pneumonia, the "digital divide" has been brought to people's attention. Older people are facing an "information divide" and even a "digital divide". The 47th Statistical Report on the Development of the Internet in China shows that by December 2020, China's Internet users aged 60 and above had exceeded 100 million person-times. Since the outbreak of COVID-19, the news of the elderly being hindered from traveling without health code has attracted wide attention. With the rapid development of digital information, Chinese society has to face the phenomenon of the aging "digital divide".

Since 2020, COVID-19 has accelerated the development of digital information technology. Based on this, the phenomenon of digital divide between the elderly should also cause enough attention of the society.

\section{THE "DIGITAL DIVIDE" AND SOCIAL INCLUSION}

The "digital divide" is usually defined as the difference between those who can and cannot use information and communication technologies. Its essence is the imbalance in the popularization and application of emerging digital, information and communication technologies represented by the Internet[1]. The digital divide phenomenon not only exists in different regions, industries and classes, but is also influenced by the age structure. The digital divide is a new imbalance that accompanies information and communication technologies, and its effective bridging can narrow the gap between rich and poor and promote social equity.

Social integration theory originated from western scholars' research on international migration, and has become a widely used concept in public administration, demography, sociology and other disciplines. In the context of the Internet, scholars emphasize that the social integration of the elderly has the following characteristics: social integration is a dynamic process, affected by technological development, the elderly's own social capital and other factors; The digital social integration of the elderly is not only a goal, but also a means to achieve online and offline social integration. The social integration of the elderly is multi-dimensional, covering social and psychological integration, as well as intergenerational cultural integration[2]. Based on relevant studies and the development status of digital information in today's society, this paper believes that bridging the "digital divide" of the elderly is conducive to promoting the social integration of this group and is of great significance to the construction of an age-friendly society. 


\section{CAUSES OF THE "DIGITAL DIVIDE" AMONG THE ELDERLY}

The phenomenon of "digital divide" in the elderly group is affected by internal and partial factors, the internal factor mainly refers to their own quality, the external factor mainly refers to the social environment.

\subsection{The Physical Restriction}

The frequency of Internet use is negatively correlated with age, with older people having a lower percentage of Internet users than younger people. Older people feel that being older prevents them from embracing and using new technology. Geriatrics research shows that the decline of function in the aging process mainly includes the sensation and perception level, the elderly's visual sensitivity decreased, narrow field of vision, not adapt to strong light, hearing reduced, sensory sensitivity decreased, finger and joint flexibility decreased, is not beneficial to the operation of keyboard and mouse. These physical barriers to aging have a significant impact on older people's use of the Internet[3]. At the same time, the elderly are more likely to be influenced by ideas, living habits and education levels, and tend to use traditional media.

\subsection{Educational Level Limit}

The level of education is also an important factor in creating the digital divide among the elderly. The penetration of traditional literacy skills decreases with age. Disabilities in reading or writing may also contribute to the digital divide among the elderly, while older Internet users with higher education are more engaged in cognitive-enhancing activities such as information and news[4]. Therefore, the ability to access and use Internet media varies with the educational status.

\subsection{Stigmatizing The Image Of Elderly Media}

The network media also has deviation in the image communication of the elderly, and the elderly group is more presented as negative media images. The anxiety and discrimination of network media groups on the participation of the elderly in media culture reflects the differences and contradictions of media choices of different groups, and also reflects the value conflicts between different groups of media culture, that is, the youth media culture treats the elderly media culture differently. Related news media reports on the "elderly" related events are easy to become the focus of public opinion, and the negative image of the elderly in the network communication has led to the stigma of the elderly group.

\subsection{Lack of continued social support}

The family environment will provide conditions and support for the elderly to access digital information and continue to socialize. Wei Dawei's survey found that among the elderly who do not use the Internet, about 30 percent of the elderly finally give up because their family members do not support them[5].The boundary between public space and private space in the Internet is blurred. Mobile social platforms extend the real interpersonal network and provide opportunities to meet strangers. Due to the blurring of the boundary between "real communication" and "virtual communication", the elderly with Internet use experience may face information security problems, and thus have anxiety about Internet social communication and information[6]. Perfect support for continued socialization environment will greatly reduce the barriers for the elderly to access information technology and improve their utilization rate.

\section{THE NECESSITY OF BRIDGING THE "DIGITAL DIVIDE" OF THE ELDERLY AND PROMOTING THEIR SOCIAL INTERGRATION}

\subsection{The Needs Of The Elderly For "Digital Life"}

In the process of rapid global digitalization, the elderly have begun to actively integrate into the Internet and become the focus of attention in the field of mobile Internet. The use of Internet-based social media can meet the diverse needs of today's elderly to varying degrees, and gradually guide them from the era of traditional media to the new mobile smart media environment.

\subsubsection{Emotional Needs}

The elderly are a relatively vulnerable group in the social structure. Older people are more emotionally dependent on relatives and peer groups for support. With the help of other We Chat and social media, it is easy to chat with family and friends. At the same time, in the process of learning to use We Chat and other social media, the elderly need guidance from family members, which can enhance their interaction in daily life. Media based on digital information technology has become an effective tool to maintain emotions, meet the emotional needs of the elderly, obtain happiness and reduce loneliness.

\subsubsection{Life Needs}

Using the network to solve problems has become an indispensable skill for social development and life. The Internet brings convenience to users, but it also brings 
negative effects to the elderly who are not familiar with the Internet. News reports show that some elderly people are unable to use the Internet to buy train tickets or pay for shopping. Therefore, in order to adapt to the current life and media environment, more and more elderly people are actively learning to use functions such as smart phones, social media and online payment under the guidance of their families. It can be seen that the elderly have the will to keep up with the development of society and The Times through learning.

\subsubsection{Social Needs}

For retired old people, with the decrease of social participation, it is easy to have loneliness and cause more social problems. The social nature of social media can not only help the elderly to maintain communication with family members, but also expand their social scope and enhance the social interaction between people to a certain extent. Keeping up with the trend of social development and mastering more information will help narrow the information gap between generations and promote intergenerational communication.

\subsection{The Need For Building An Age-friendly Society}

In recent years, the number of the elderly population in China is increasing, the degree of social aging is increasing, and the social problems caused by this are becoming more and more obvious. During the outbreak of novel coronavirus pneumonia, the elderly became the main target for the prevention and control of the epidemic because of their poor physical fitness and many basic diseases. However, due to a number of barriers in receiving prevention and control information, the elderly have a poor awareness of the epidemic, resulting in poor self-protection awareness, travel disruption, impact on life, purchase of epidemic prevention supplies, posing a great threat to health. Increasing the use of Internet devices among the elderly will also be an important task in building an aging society.

\subsection{Promoting The Economic Participation Of The Younger And Older Groups}

To a certain extent, bridging the digital divide and unimpeded access to information for the elderly can promote their continued employment. As an important part of active aging, the employment participation behavior of the elderly can cope with the increasingly serious labor shortage, and has a positive significance for relieving the pressure of supporting the elderly. Adapting to the development of digital information society and making full use of Internet information can help the elderly to return to the labor market, make full use of their rich experience and skills, and make corresponding contributions to social construction.

\subsection{The Need To Normalize Epidemic Prevention And Control}

During the epidemic period, Internet government affairs, online consultation, travel health code and other measures were widely used. In the post-epidemic era, epidemic prevention and control measures based on digital information technology have become a normal phenomenon and have been applied in many areas of society. Due to the low Internet and smartphone usage, the elderly may face difficulties in getting in and out of the community, taking public transport, shopping in supermarkets, registering online and handling affairs online, and their daily life is greatly affected. The above phenomenon is the epitome of the information weakness of the elderly in the face of major events such as the COVID-19 epidemic. Helping the elderly to access Internet media and adapt to the development trend of the information society can reduce various travel barriers faced by the elderly, facilitate their social mobility and reduce the impact of the epidemic on them, which is also of great significance for epidemic prevention and control.

\section{CONCLUSION}

COVID-19 highlights the advantages of digital technology and the digital economy in social governance and economic development, and drives a wave of digital change. In the process of accelerating the development of the digital economy, it is also becoming increasingly important to pay attention to the "digital divide" among the elderly.

\section{ACKNOWLEDGMENTS}

Supported by National Social Science Foundation of China Key Project "Local practice of long-term care insurance system for disabled elderly" (Project No.: 17BRK015)

\section{REFERENCES}

[1] Hu Angang. The new global wealth gap and the growing digital divide. [J]. Social Sciences in China,2002 (3) : 34-48.

[2] Yue Zhongshan, Du Haifeng, Li Shuzhuo, et al. The concept, theory and application of contemporary western social integration research [J]. Journal of Public Administration, 2009(4) : 114-120.

[3] Floria Kohlbach, Cornelius Hestadt. Silver hair market phenomenon -- aging society marketing and innovative thinking [M]. Hu Zhongyan, Lu Jinting, 
Trans. Dalian: Dongbei University of Finance and Economics Press, 2016:47-73.

[4] ALEXANDER J, VAND. A nuanced understanding of internet use and non-use among the elderly [J]. European journal of communication, 2015, 30( 2) : 171-187.

[5] Wei Dawei. Research about the obstacles of use of the Internet to the elderly in China from the perspective of the digital divide. [D] .Wu Han: Wuhan Textile University ,2012: 28-30.

[6] WAGNER N, HASSANEINM K , HEAD M.Computer-use by older adults: a multidisciplinary review [J]. Computers in human behavior, 2010, 26( 5) : 870-882. 